\title{
ANALYSIS OF LYSATE PLATELET-RICH FIBRIN EFFECTS ON HUMAN DENTAL PULP STEM CELL DIFFERENTIATION THROUGH DENTIN SIALOPHOSPHOPROTEIN EXPRESSION
}

\author{
SILVIANA SWASTININGTYAS ${ }^{1}$, ANGGRAINI MARGONO ${ }^{2 *}$, DINI ASRIANTI ${ }^{2}$, RUNY OKTAYANI $^{1}$, INDAH YULIANTO ${ }^{3}$ \\ ${ }^{1}$ Conservative Dentistry Residency Program, Faculty of Dentistry, Universitas Indonesia, Jakarta, Indonesia. ${ }^{2}$ Department of Conservative \\ Dentistry, Faculty of Dentistry, Universitas Indonesia, Jakarta, Indonesia. ${ }^{3}$ Department of Dermatology, Faculty of Medicine, Universitas \\ Sebelas Maret, Surakarta, Central Java, Indonesia. Email: margonodewi@yahoo.com
}

Received: 23 July 2019, Revised: 28 February 2020, Accepted: 01 June 2020

ABSTRACT

Objective: In vitro, the culture media in which human dental pulp stem cells (hDPSCs) are grown are supplemented with specific growth factors that induce cell cycle entry and differentiation. Lysate platelet-rich fibrin (L-PRF) is a unique and stable growth factor supplement produced from platelets lysed by freezing-thawing. In this study, we aimed to analyze the potential effects of L-PRF on hDPSC differentiation.

Methods: We divided hDPSCs isolated from human third molars at the second passage into five culture media groups treated with $1 \%$, $5 \%$, $10 \%$, and $25 \%$ L-PRF or $10 \%$ fetal bovine serum (control). After 7 days, we evaluated hDPSC differentiation using an enzyme-linked immunosorbent assay specific for dentin sialophosphoprotein and Alizarin-Red staining.

Results: None of our analyses revealed any significant differences between the L-PRF- and control-treated cells.

Conclusion: L-PRF could potentially induce the differentiation of hDPSCs in vitro.

Keywords: Lysate platelet-rich fibrin, Differentiation, Human dental pulp stem cells, Dentin sialophosphoprotein.

(C) 2020 The Authors. Published by Innovare Academic Sciences Pvt Ltd. This is an open access article under the CC BY license (http://creativecommons. org/licenses/by/4. 0/) DOI: http://dx.doi.org/10.22159/ijap.2020.v12s2.0P-43

\section{INTRODUCTION}

Regenerative endodontic procedures (REPs) comprise a type of bioengineering therapies that aim to restore the physiological functions of the dental pulp [1]. This alternative dental bioengineering approach consists of three classic elements, namely, dental stem cells, signal molecules, and scaffolds, and two new elements, namely, stem cell niches and survival/trophic factors [1]. The isolation of highly proliferative pulp cell subpopulations with multilineage (including odontoblast) differentiation capacities has elucidated the complex mechanisms underlying the healing capacity of the dental pulp [2]. Dental stem cells and their daughter cells grow and differentiate in response to growth factors released by the surrounding tooth microenvironment and dental stem cell niches. During REP therapies, the triad aims to reconstruct these microenvironments [1]. For example, growth factors (TGF- $\beta 1$, BMP-7, FGF-2, IGF-1 and IGF-2, $\mathrm{NGF}$, and GDNF), extracellular matrix molecules (dentin sialoprotein [DSP], dentin phosphoprotein [DPP], BSP, DMP-1, and dentin sialophosphoprotein [DSPP]), and both anti- and pro-inflammatory chemokines and cytokines (TNF- $\alpha$, IL-1, IL- 6 , and IL-10) are some dentin-derived signal molecules [1,3] that regulate cell-cell interactions and cell proliferation and differentiation during tooth development [1]. Moreover, scaffolds are biocompatible structures that support cell growth and provide a suitable environment for tissue formation. A good scaffold should enable cell attachment, proliferation, migration, and differentiation and provide mechanical support for extracellular matrix generation [4].

In 2000, Choukroun et al. developed a second-generation platelet concentrate that did not utilize anticoagulation factors [5]. This novel platelet concentrate could be harvested from the upper layers of centrifugation tubes after a single 12-min centrifugation cycle at $2700 \mathrm{rpm}(750 \mathrm{~g})$ [6]. This concentrate was shown to release growth factors slowly over a prolonged period of 7-14 days and is thus superior to PRP, which releases growth factors rapidly over a period of 7-14 h [4]. A modification of the preparation settings based on a previously identified low-speed centrifugation concept represented an initial step in the reduction of the applied relative centrifugation force, which was accompanied by a mild increase in the centrifugation time and resulted in a so-called advanced PRF [5]. In contrast, lysate plateletrich fibrin (L-PRF) was then prepared by subjecting peripheral blood to platelet lysis through different freezing-thawing cycles and collecting the released growth factors [7].

According to Niwa et al., the expression of DSPP mRNA increases with the presence of TGF $\beta$ on day 7 [8]. Cell proliferation will slow and stop while the increase in TGF $\beta$ expression on day 7 and cell differentiation can begin. This observation was confirmed by Saeed et al. who evaluated the differentiation of human dental pulp stem cells (hDPSCs) in response to several concentrations of PRF exudate $(1 \%, 5 \%, 10 \%$, and $20 \%)$ in on day 7 and found that $1 \%$ PRF exudate exhibited the strongest osteodifferentiation ability through AlizarinRed staining and calcium deposit analyses [4]. In another study, Abuarqoub et al. supplemented odontogenic differentiation media with different concentrations of lysate platelets derived from PRP and evaluated hDPSCs and SCAP on days 1, 9, 15, and 21 using an enzymelinked immunosorbent assay (ELISA); here, a 5\% PRP concentration was identified as superior according to the DSPP level on day 15 and the differentiation pattern on day 21 [7]. One can conclude that, although the concentration of platelet concentrate in culture can influence cell differentiation, a higher concentration does not always result in better differentiation.

\section{METHODS}

This study was approved by the Ethics Committee of the Faculty of Dentistry, Universitas Indonesia (no. 35/ethical approval/ FKGUI/V/2018, no. protocol: 090250218). Six-third molars were collected from healthy human donors aged 19-35 years with no history of alcohol consumption or smoking at the oral surgery department, 
Faculty of Dentistry, Universitas Indonesia. The teeth were transferred to the cell culture laboratory, where stem cells were isolated from the pulp tissues. Briefly, each tooth was cut around the cementoenamel junction, after which the pulp tissue was gently removed from the chambers, sliced into $0.5-1.0 \mathrm{~mm}$ sections, and diluted in an enzyme solution that consisted of $3.0 \mathrm{mg} / \mathrm{mL}$ collagenase Type I and $4.0 \mathrm{mg} / \mathrm{mL}$ dispase (Sigma-Aldrich, St. Louis, MO, USA) for $30-60 \mathrm{~min}$ at $37^{\circ} \mathrm{C}$. The resulting mixture was cultured in $3 \mathrm{~mL}$ of Dulbecco's modified Eagle's medium (DMEM) supplemented with 10\% fetal bovine serum (FBS; HyClone), $100 \mathrm{U} / \mathrm{mL}$ penicillin G (Roche, Basel, Switzerland), and $100 \mathrm{mg} / \mathrm{mL}$ streptomycin (Roche) and incubated in an atmosphere of $5 \% \mathrm{CO}_{2}$ at $37^{\circ} \mathrm{C}$. Once the cell cultures reached $80 \%$ confluency, single cell clones were isolated by limiting dilution. In this study, all cells used in further experiments were of the second or third passage. At the second passage, hDPSCs were divided into five culture media groups: $1 \%, 5 \%, 10 \%$, and $25 \%$ L-PRF, and 10\% FBS (control). Each group contained six biological replicates, and all cells were treated as indicated after $24 \mathrm{~h}$ of serum starvation according to a previously published method [9].

L-PRF was produced from 5 -ml blood samples collected from volunteers with no history of smoking, alcohol consumption, or anticoagulant use. All participants provided signed informed consent. Briefly, the blood samples were collected into 5 -ml vacuum blood collection tubes without anticoagulant and were immediately centrifuged at $2700 \mathrm{rpm}$ for $12 \mathrm{~min}$. A white clot comprises PRF formed in the middle of each tube between the plasma and RBC layers. The PRF clot was removed using a sterile forceps and incubated in an empty tube at $4^{\circ} \mathrm{C}$ for $24 \mathrm{~h}$ to produce L-PRF according to the previously described method. Once the residual fibrin had attached to the bottoms of the tubes, the supernatant was aspirated, transferred to a 2-ml Eppendorf tube, and stored at $-20^{\circ} \mathrm{C}$. Subsequently, the supernatant was diluted in DMEM to concentrations of $1 \%, 5 \%, 10 \%$, and $25 \%$ L-PRF.

Osteogenic differentiation media comprised nutritional media (DMEM-F12), $50 \mu \mathrm{g} / \mathrm{ml}$ of L-ascorbic acid 2-phosphate, $10 \mathrm{mM}$ $\beta$-glycerophosphate sodium salt, $10 \mu \mathrm{M}$ dexamethasone, and antibiotics $(100 \mathrm{mg} / \mathrm{ml}$ streptomycin and $100 \mu / \mathrm{ml}$ penicillin G). For osteogenic differentiation, four wells per 6-well plate were used. A total of $10^{5}$ cells were cultured in each well in complete media until the cells reached nearly $70 \%$ confluence. After $24 \mathrm{~h}$ of serum starvation, the cells were maintained in osteogenic differentiation media containing different concentrations of L-PRF. Mineralized nodule formation was detected and quantified after 7 days by DSPP expression using ELISA reader and Alizarin-Red-based assay.
The results from each group and control were analyzed using the Kruskal-Wallis test and post hoc Mann-Whitney test with $\mathrm{p}=0.5$.

\section{RESULTS}

We first used Alizarin-Red staining to investigate the mineralization potential of hDPSCs undergoing osteogenic differentiation. In all wells, Alizarin-Red staining clearly identified mineralized nodules. However, there were obvious differences in the amounts of mineralization among the different groups. Particularly, the mineralized nodules progressively increased with $1 \% \mathrm{~L}-\mathrm{PRF}$ at the day 7 of culture in osteogenic medium (Fig. 1). The best pattern of differentiation was noted with $1 \% \mathrm{~L}-\mathrm{PRF}$, followed by $5 \%, 10 \%$ and $25 \% \mathrm{~L}-\mathrm{PRF}$.

\section{DISCUSSION}

Stem cells naturally secrete bioactive molecules that support tissue regeneration. These bioactive molecules, or secretomes, have biological functions in cell development, proliferation, differentiation, apoptosis, immune system modulation, adhesion, and angiogenesis. This is used in the cell free-based therapy approach so that stem cell transplants are not needed to root canals [10]. Stem cells can proliferate and differentiate in response to the growth factors released by the surrounding environment, such as the culture media supplements provided in vitro [1].

Different stem cell starvation and activation protocols are used in in vitro studies. Previously, researchers have induced hDPSC differentiation through serum starvation in culture media containing $1 \%$ FBS, and this process may occur for some period of time. On nutrient deprivation, cells typically exit the cell cycle and implement survival mechanisms based on the minimal usage of energy and other resources. This elimination of serum from the culture medium provides reproducible experimental conditions [11]. Although FBS is a universal growth supplement used in cell and tissue culture media, serious ethical concerns have been raised regarding the welfare of the donor fetuses and the potential for adverse contaminants, such as endotoxin, mycoplasma, viral contaminants, or prion proteins [4]. Therefore, this study aimed to investigate an alternative to FBS that would promote hDPSC differentiation in vitro and emphasize the potential use of L-PRF in this capacity.

In this study cell, we measured cell differentiation using Alizarin-Red staining and DSPP expression and determined that the mineralized deposits were most clearly formed in the culture containing $1 \% \mathrm{~L}-\mathrm{PRF}$,

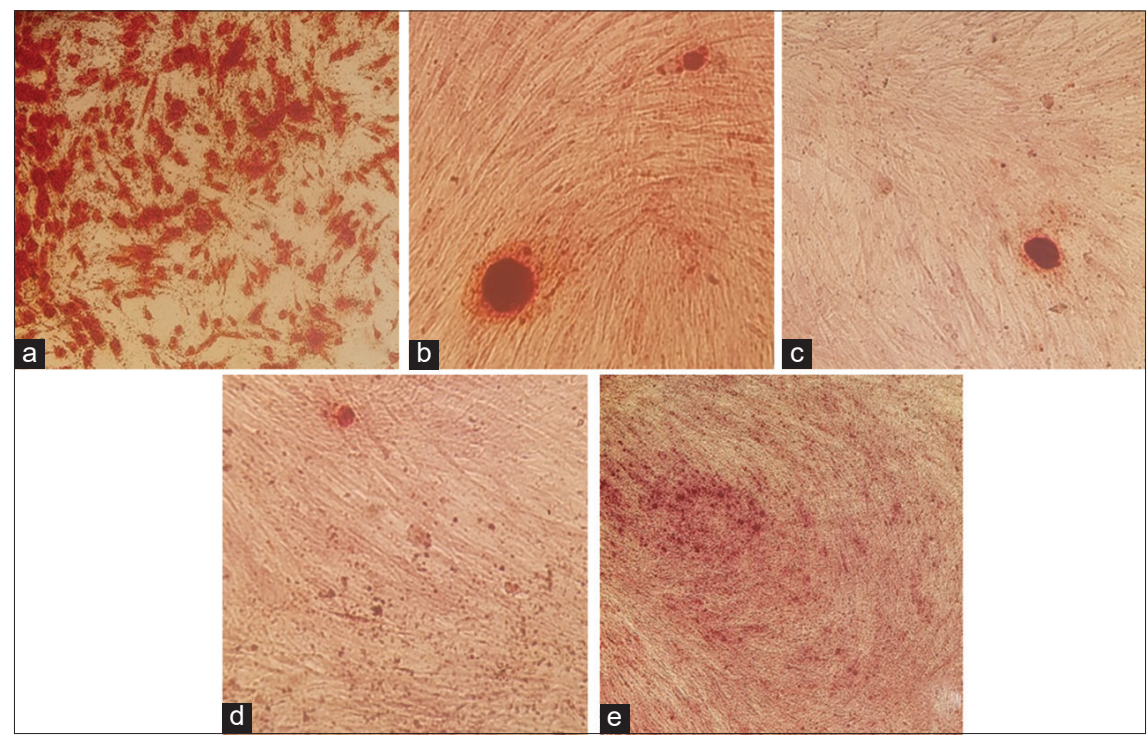

Fig. 1: Identification of mineralized deposits in cells grown in the osteogenic medium by Alizarin-Red S staining on day 7 . Alizarin-Red $S$ staining of human dental pulp stem cells as viewed using an inverted light microscope at $\times 10$. (a) $10 \%$ Fetal bovine serum (positive control); (b) 1\% Lysate platelet-rich fibrin (L-PRF); (c) 5\% L-PRF; (d) 10\% L-PRF; (e) 25\% L-PRF 


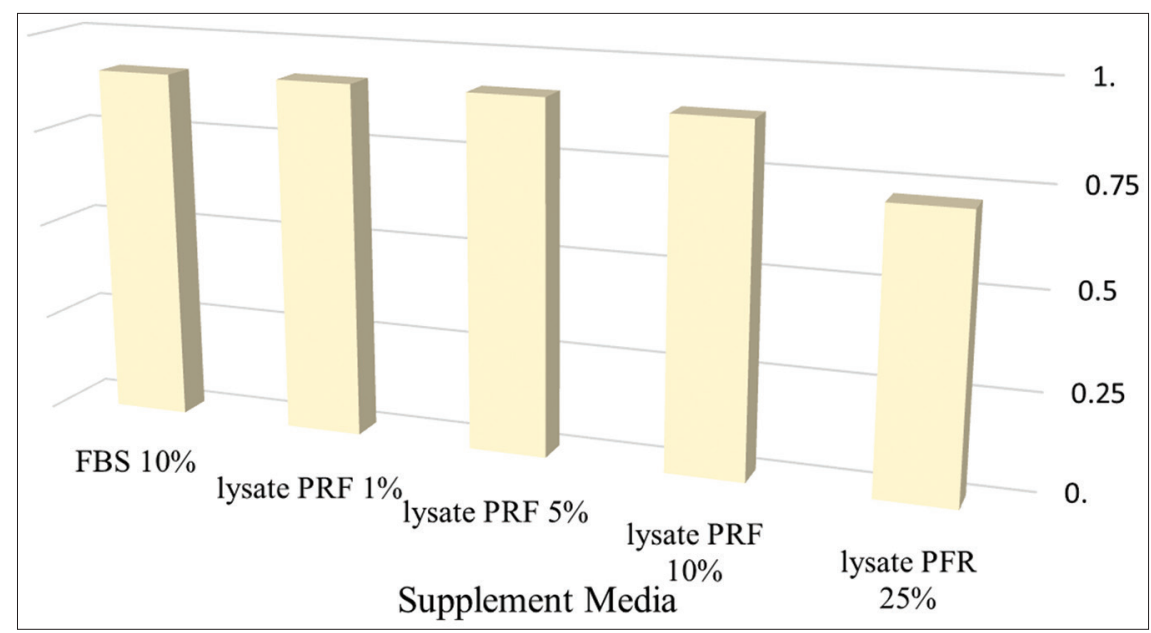

Fig. 2: Analysis of mean dentin sialophosphoprotein expression levels in response to various concentrations of lysate platelet-rich fibrin on day 7

Table 1: Analysis of DSPP expression in response to various concentrations of L-PRF at day 7

\begin{tabular}{lllll}
\hline Supplement media & n & Median & Min-Max & p \\
\hline FBS 10\% (control) & 2 & 0.907 & $0.890-0.925$ & 0.074 \\
L-PRF 1\% & 3 & 0.911 & $0.900-0.926$ & \\
L-PRF 5\% & 3 & 0.910 & $0.890-0.931$ & \\
L-PRF 10\% & 3 & 0.890 & $0.880-0.901$ & \\
L-PRF 25\% & 3 & 0.685 & $0.615-0.854$ & \\
\hline
\end{tabular}

*Kruskal-Wallis test, $\mathrm{p}>0.05$

consistent with the results of a previous study by Saeed et al., in which a 1\% PRF exudate yielded the highest osteodifferentiation ability on day 7 [4]. Moreover, we showed that although the differences in DSPP expression between the different L-PRF concentrations were not significant, 1\% L-PRF induced a higher level of DSPP expression than $25 \%$ L-PRF. We attribute this result to the inflammatory mediators released by other immune cells present in PRF, which could potentially inhibit cell differentiation $[12,13]$. This mechanism would explain why a higher L-PRF concentration does not always provide superior induction of differentiation.

The results of the DSPP ELISA analysis were consistent with those of Alizarin-Red staining, which most clearly revealed red nodules with $1 \%$ L-PRF. DSPP is a biomineralization of predentin into a dentin-specific marker secreted by the odontoblast. DSPP consists of DSP, dentin glycoprotein, and DPP; the former supports the growth, migration, and odontogenic differentiation of pulp cells, while the latter plays a role in dentine mineralization by binding to collagen and initiating hydroxyapatite crystal formation [3,14]. Therefore, DSPP is an important non-collagen protein in dentine that cannot be found in other tissues and is thus a phenotypic marker of odontoblast activity $[15,16]$. Both our analyses led us to conclude that the highest odontogenic differentiation in vitro occurred in culture media supplemented with $1 \% \mathrm{~L}-\mathrm{PRF}$. The four tested L-PRF supplement concentrations $(1 \%, 5 \%$, $10 \%$, and $25 \%$ ) clearly induced the differentiation of hDPSCs in vitro. However, we did not observe a significant difference between these concentrations. These findings are in contrast to those of Asri et al., [17] who recently reported that the highest level of hDPSC proliferation was induced by $25 \% \mathrm{~L}-\mathrm{PRF}$. Therefore, in a cell-free therapy, the use of $25 \%$ PRF lysate as a growth medium is recommended to support the proliferation and differentiation of hDPSCs.

\section{CONCLUSION}

Quantitatively, our ELISA analysis of the levels of DSPP produced by hDPSCs in response to different concentrations of L-PRF did not reveal any significant differences (Kruskal-Wallis test; Table 1). However, the graph in Fig. 2 demonstrates that the highest and lowest mean DSPP levels were observed at $1 \%$ and $25 \%$ L-PRF, respectively (0.912 and 0.718 , respectively).

\section{ACKNOWLEDGMENTS}

We thank our colleagues Yanni Dirgantara S.Si and Emilia Rahmadaniah Utami, S.Si, from the ProSTEM Laboratory, and our collaborators for their excellent experimental assistance and discussions.

\section{REFERENCES}

1. Mari-Beffa M, Segura-Egea JJ, Diaz-Cuenca A. Regenerative endodontic procedures: A perspective from stem cell niche biology. J Endod 2017;43:52-62.

2. Conde MC, Chisini LA, Demarco FF, Nör JE, Casagrande L, Tarquinio SB. Stem cell-based pulp tissue engineering: Variables enrolled in translation from the bench to the bedside, a systematic review of literature. Int Endod J 2016;49:543-50.

3. da Rosa WL, da Silva TM, Galarça AD, Piva E, da Silva AF. Disclosing the physiology of dental pulp for vital pulp therapy. Int Endod J 2018;51:829-46.

4. Saeed MA, El-Rahman MA, Helal ME, Zaher AR, Grawish ME. Efficacy of human platelet rich fibrin exudate vs fetal bovine serum on proliferation and differentiation of dental pulp stem cells. Int J Stem Cell 2017;10:38-47.

5. El Bagdadi K, Kubesch A, Yu X, Al-Maawi S, Orlowska A, Dias A, et al. Reduction of relative centrifugal forces increases growth factor release within solid platelet-rich-fibrin (PRF)-based matrices: A proof of concept of LSCC (low speed centrifugation concept). Eur J Trauma Emerg Surg 2019;45:467-79.

6. Choukroun J, Aalam AA, Miron RJ. Platelet rich fibrin PRF and regenerative medicine: The low-speed concept. In: Tatullo M, editor. MSCs and Innovative Biomaterials in Dentistry. Cham: Humana Press; 2017. p. 21-42.

7. Abuarqoub D, Awidi A, Abuharfeil N. Comparison of osteo/ odontogenic differentiation of human adult dental pulp stem cells and stem cells from apical papilla in the presence of platelet lysate. Arch Oral Biol 2015;60:1545-53.

8. Niwa T, Yamakoshi Y, Yamazaki H, Karakida T, Chiba R, Hu JC, et al. The dynamics of TGF- $\beta$ in dental pulp, odontoblasts and dentin. Sci Rep 2018;8:4450.

9. Marsa RD, Asrianti D, Margono A. The efficacy of platelet-rich fibrin lysate (PRF-L) for fibroblast cell proliferation. J Int Dent Med Res 2017;10:809-813.

10. Chaparro O, Linero I. Regenerative Medicine: A New Paradigm in Bone Regeneration. Tamil Nadu: Intech; 2016. p. 253-74.

11. Aghababazadeh M, Kerachian MA. Cell fasting cellular response and application of serum starvation. J Fasting Health 2014;2:147-50.

12. Hong S, Chen W, Jiang B. A comparative evaluation of concentrated growth factor and platelet-rich fibrin on the proliferation, migration, and differentiation of human stem cells of the apical papilla. J Endod 
2018;44:1-7.

13. Masuki H, Okudera T, Watanebe T, Suzuki M, Nishiyama K. Growth factor and pro-inflammatory cytokine contents in platelet-rich plasma (PRP), plasma rich in growth factors (PRGF), advanced platelet-rich fibrin (A-PRF), and concentrated growth factors (CGF). Int J Implant Dent 2016;2:1-6.

14. Narayanan K, Srinivas R, Ramachandran A, Hao J, Quinn B, George A. Differentiation of embryonic mesenchymal cells to odontoblast-like cells by overexpression of dentin matrix protein 1 . PNAS 2001;98:4516-21.
15. Emilia E, Neelakantan P. Biomarkers in the dentin-pulp complex: Role in health and disease. J Clin Pediatr Dent 2015;39:94-9.

16. Suzuki S, Haruyama N, Nishimura F, Kulkarni AB. Dentin sialophosphoprotein and dentin matrix protein-1: Two highly phosphorylated proteins in mineralized tissues. Arch Oral Biol 2012;57:1165-75.

17. Asri SR, Setiati HD, Asrianti D, Margono A, Usman M, Yulianto I Optimum concentration of platelet-rich fibrin lysate for human dental pulp stem cells culture medium. J Int Dent Med Res 2019;12:105-10. 